\title{
Gender- and Age-Specific Associations between Visceral Obesity and Renal Function Impairment
}

\author{
Yu-Chung Tsao ${ }^{a-c}$ Jau-Yuan Chen ${ }^{b, c}$ Wei-Chung Yeh ${ }^{b, c}$ Wen- \\ Cheng Lib, d, e \\ a Department of Occupational Medicine, Chang Gung Memorial Hospital, Taoyuan, Taiwan; \\ ${ }^{b}$ Department of Family Medicine, Chang Gung Memorial Hospital, Taoyuan, Taiwan;

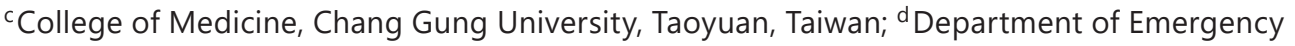

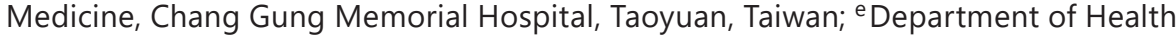 \\ Management, Xiamen Chang-Gung Hospital, Xiamen, PR China
}

\section{Keywords}

Visceral obesity $\cdot$ Peripheral obesity $\cdot$ Kidney disease $\cdot$ Gender

\begin{abstract}
Objective: Although obesity is associated with an increased risk of chronic kidney disease, this trend becomes nonsignificant following adjustment for cardiovascular risk factors. The present study aims to investigate whether visceral obesity is independently associated with renal function impairment. Method: The medical records of 14,529 male and 10,561 female Chinese adults undergoing health check-ups during 2013-2015 were retrospectively collected. The baseline characteristics, including the degree of visceral fat and the percentage of body fat, were compared. The association between study groups and renal function impairment was investigated using regression models adjusted for confounding factors. Results: All variables differed significantly among non-obese, peripheral, and central type obese subjects, both younger and older, and of both genders, except for hsCRP in older male subjects ( $p=$ 0.053 ) and eGFR in older female subjects $(p=0.098)$. Unadjusted univariate analysis showed that central obesity contributed significantly to renal function impairment in all age groups and in both genders. After adjusting for possible confounding factors, only central obesity was found to be an independent factor of renal function impairment in all groups, except for men under 45 years of age. Conclusion: Visceral obesity is independently associated with renal function impairment in all ages and both genders, except for males younger than 45 years.
\end{abstract}




\section{Introduction}

Chronic kidney disease (CKD) is characterized by a reduced estimated glomerular filtration rate (eGFR), increased urinary albumin excretion, or both. It constitutes an increasingly common public health issue, with an estimated worldwide prevalence of 8-16\%, and its complications include increased all-cause and cardiovascular mortality, kidney disease progression, acute kidney injury, cognitive decline, anemia, mineral and bone disorders, and fractures [1].

The literature on regional adipose tissue distribution and metabolism has flourished over the past decades, establishing that the proportion of abdominal adipose tissue is a key correlate, and perhaps a driver, of overweight and obesity-associated health risks [2]. The accumulation of visceral fat is an important component of the metabolic syndrome, which encompasses various metabolic disorders such as glucose intolerance, dyslipidemia, and hypertension, and is associated with atherosclerotic cardiovascular diseases [3]. Obesity is also thought to be associated with an increased risk for stage $3 \mathrm{CKD}$, although that trend becomes nonsignificant after adjustment for known cardiovascular disease risk factors. The relationship between obesity and stage 3 CKD may be mediated by risk factors of cardiovascular disease [4]. The visceral adipose tissue (VAT) is known to have a high lipolytic rate, generating large amounts of free fatty acids which are delivered to the liver, causing increased hepatic glucose production, hyperinsulinemia, and metabolic syndrome [5].

A previous study suggested that visceral fat accumulation, estimated using bioelectric impedance analysis, is associated with an increased urinary albumin-creatinine ratio (ACR) [6]. Another study found that obesity was significantly related to an increased albumin excretion rate, irrespective of the obesity type. A significant difference in the risk of renal malfunction was observed in individuals with a similar percentage but different distribution of total body fat (BF), with the central fat pattern comprising the greater risk [7]. However, age and gender-related differences remain unclear.

The aim of the present study was to investigate whether visceral obesity is independently associated with renal function and whether fat tissue measurement is useful to detect renal impairments. Additionally, age and gender-related differences were investigated.

\section{Methods}

The medical records of Chinese adults (aged $\geq 18$ years) undergoing health check-ups during 20132015 at the Chang Gung Memorial Hospital were retrospectively collected. Subjects with incomplete data, a history of any chronic disease or medications likely to affect the metabolic status or kidney function (e.g., thyroid or hypothalamic disease, adrenal gland disease, renal cancer, status postrenal transplantation, glomerulonephritis, nephritic syndrome, hepatocellular carcinoma, cirrhosis, the use of diuretics, thyroid medication, or renal replacement therapy), and pregnant women were excluded from this study. A total of 14,529 males and 10,561 females were included in the analysis. The study was approved by the Institutional Review Board of the Chang Gung Memorial Hospital and was performed in accordance with the Helsinki Declaration.

Body height and weight were measured using calibrated meters and scales. The body mass index (BMI) was calculated using the following formula: body weight $(\mathrm{kg}) /([\text { height in } \mathrm{m}])^{2}$. Blood pressure was measured after a 10-min rest period, with the subject in a seated position, using an automated sphygmomanometer placed on the subject's right arm. The mean arterial pressure (MAP) was estimated using the following equation: $(2 / 3) \cdot$ diastolic pressure $+(1 / 3) \cdot$ systolic pressure. Subjects were requested to fast for a minimum of $12 \mathrm{~h}$ and to avoid a high-fat diet or alcohol consumption for at least $24 \mathrm{~h}$ prior to phlebotomy. Fresh urine samples were used for urinary albumin and creatinine measurement, performed using a biochemical test (UniCel ${ }^{\circledR}$ DxC 800 MA\&CREA. Reagent). Spot urine ACR were calculated for all participants. Venous blood samples were obtained at 5:30-11:00 a.m. and stored in a $4{ }^{\circ} \mathrm{C}$ refrigerator, prior to analysis in the hospital 
Tsao et al.: Association between Visceral Obesity and Renal Function Impairment

laboratory. Clinical chemistry workups included fasting plasma glucose (mmol/L), serum creatinine (sCr; $\mu \mathrm{mol} / \mathrm{L}$ ), total cholesterol (TC; mmol/L), low-density lipoprotein (LDL; mmol/L), high-density lipoprotein (HDL; mmol/L), triglyceride (TG; mmol/L), and high-sensitivity C-reactive protein (hsCRP; $\mu \mathrm{g} / \mathrm{mL}$ ), which were measured using a biochemical auto-analyzer (DxC 800; Beckman Coulter UniCel ${ }^{\circledR}$ DxC SYNCHRON ${ }^{\circledR}$, Ireland). Blood tests were carried out in accordance with the hospital's laboratory SOP, which was accredited by the College of American Pathologists.

The total percent body and visceral fat degree were measured by bioelectric impedance analysis, using a portable stand-on analyzer (InBody 3.0 model; Biospace, Seoul, Korea) placed on a hard-level surface. To ensure measurement accuracy, the subjects were asked not to do physical exercise or consume alcohol for at least $24 \mathrm{~h}$ prior to the examinations. The gender-specific percentage of body fat (BF\%) cutoffs according to the definitions of the Taiwan Medical Association for the Study of Obesity (http://www.obesity.org.tw/) were as follows: (1) low BF\%: men $<17 \%$ and women $<20 \%$; (2) normal BF\%: men 17-23\% and women 20-27\%; and (3) high BF\%: men $>23 \%$ and women $>27 \%$. In the present study, central obese type was defined as $\mathrm{BF} \%>23 \%$ with a visceral fat degree $>10$ in males and $\mathrm{BF} \%>27 \%$ with visceral fat degree $>10$ in females. The non-obese type was defined as $\mathrm{BF} \% \leq 23 \%$ in males and $\leq 27 \%$ in females. The peripheral obese type was defined as $\mathrm{BF} \%>23 \%$ with a visceral fat degree $\leq 10$ in males and $\mathrm{BF} \%>27 \%$ with a visceral fat degree $\leq 10$ in females.

The modification of diet in renal disease equations for Chinese patients with CKD [8] were applied to estimate the eGFR as follows: eGFR (measured in $\left.\mathrm{mL} / \mathrm{min} / 1.73 \mathrm{~m}^{2}\right)=175 \times(\mathrm{sCr})^{-1.234} \times(\text { age })^{-0.179} \times 0.79$ (if female). Albuminuria measured in urine was classified based on the recommended ACR value cutoffs as normoalbuminuria (ACR <30 mg/g Cr), microalbuminuria (ACR: 30-299 mg/g Cr), or macroalbuminuria (ACR $>300 \mathrm{mg} / \mathrm{g} \mathrm{Cr}$ ) [9]. Following the definition of the Kidney Disease Outcomes Quality Initiative (K/DOQI) [10], each patient's CKD stage was classified based on the values of eGFR and proteinuria measurements as follows: stage 1 included participants with eGFR $\geq 90 \mathrm{~mL} / \mathrm{min} / 1.73 \mathrm{~m}^{2}$ and proteinuria; stage 2 as eGFR of $60-89 \mathrm{~mL} / \mathrm{min} / 1.73 \mathrm{~m}^{2}$ with proteinuria; stage 3 as eGFR of $30-59 \mathrm{~mL} / \mathrm{min} / 1.73 \mathrm{~m}^{2}$, stage 4 as eGFR of $15-29 \mathrm{~mL} / \mathrm{min} / 1.73 \mathrm{~m}^{2}$, and stage 5 as eGFR $<15 \mathrm{~mL} / \mathrm{min} / 1.73 \mathrm{~m}^{2}$. Patients with either CKD stage $>3$ or albuminuria were considered to have renal function impairment.

\section{Statistical Analysis}

The baseline characteristics of male and female subjects were compared using the $t$ test for continuous variables or the $\chi^{2}$ test for categorical variables. The baseline characteristics were compared among study groups composed of visceral fat degree and BF\% (i.e., non-obesity, peripheral obesity, and central obesity) using one-way analysis of variance for continuous variables and the $\chi^{2}$ test for categorical variables. A Bonferroni post hoc test was used for multiple comparisons between any specific two groups, when the overall $F$ test or $\chi^{2}$ test was significant $(p<0.05)$. The association between the study group and the renal function impairment was performed using several logistic regression models adjusted for selected confounding factors and treating the study group as an explanatory variable. All data were analyzed during 2017-2018, using SPSS 22 (IBM SPSS Inc, Chicago, IL, USA). The datasets used and/or analyzed during the current study are available from the corresponding author on reasonable request.

\section{Results}

\section{Baseline Characteristics by Gender}

Male subjects were slightly younger and exhibited higher levels of BMI, waist-to-height ratio, MAP, glucose, TC, TG, LDL, TG/HDL-C, and hsCRP, and lower levels of HDL. Male subjects also exhibited lower levels of eGFR and ACR and a lower prevalence of renal function impairment. Although the BF\% was higher in female subjects, the central obese type was more common in male subjects (Table 1).

Male Baseline Characteristics according to Visceral Fat Degree and BF\% Stratified by Age Except for the hsCRP level of older subjects $(p=0.053)$, significant differences were found for all variables among non-obese, peripheral, and central type obese subjects, both in younger and older male subjects. Males younger than 45 years with central obesity were found to have 
Tsao et al.: Association between Visceral Obesity and Renal Function Impairment

Table 1. Baseline characteristics of the study subjects by gender

\begin{tabular}{|c|c|c|c|}
\hline Characteristics & Men & Women & $p$ value \\
\hline Subjects & 14,529 & 10,561 & - \\
\hline Age, years & $43.4 \pm 11.1$ & $43.7 \pm 11.7$ & 0.027 \\
\hline \multicolumn{4}{|l|}{ Measurements } \\
\hline BMI, $\mathrm{kg} / \mathrm{m}^{2}$ & $24.5 \pm 3.3$ & $22.5 \pm 3.4$ & $<0.001$ \\
\hline Waist-to-height ratio, $\mathrm{cm} / \mathrm{cm}$ & $0.51 \pm 0.05$ & $0.49 \pm 0.06$ & $<0.001$ \\
\hline Mean arterial pressure, $\mathrm{mm} \mathrm{Hg}$ & $92.0 \pm 12.2$ & $84.5 \pm 12.6$ & $<0.001$ \\
\hline \multicolumn{4}{|l|}{ Blood chemistry data } \\
\hline Fasting glucose, mmol/L & $5.4 \pm 1.4$ & $5.2 \pm 1.0$ & $<0.001$ \\
\hline Total cholesterol, mmol/L & $5.2 \pm 1.0$ & $5.0 \pm 1.0$ & $<0.001$ \\
\hline Triglycerides, mmol/L & $1.76 \pm 1.76$ & $1.06 \pm 0.94$ & $<0.001$ \\
\hline $\mathrm{LDL}-\mathrm{C}, \mathrm{mmol} / \mathrm{L}$ & $3.3 \pm 0.9$ & $3.0 \pm 0.8$ & $<0.001$ \\
\hline HDL-C, mmol/L & $1.19 \pm 0.27$ & $1.43 \pm 0.31$ & $<0.001$ \\
\hline TG/HDL-C & $1.68 \pm 2.41$ & $0.84 \pm 1.10$ & $<0.001$ \\
\hline hsCRP, $\mu \mathrm{g} / \mathrm{mL}$ & $2.08 \pm 5.22$ & $1.45 \pm 3.65$ & $<0.001$ \\
\hline \multicolumn{4}{|l|}{ Body composition } \\
\hline $\mathrm{BF} \%$ & $21.5 \pm 5.4$ & $30.3 \pm 6.2$ & $<0.001$ \\
\hline Central obese type & $5,250(36.1)$ & $530(5.0)$ & $<0.001$ \\
\hline \multicolumn{4}{|l|}{ Renal function } \\
\hline $\mathrm{eGFR}, \mathrm{mL} / \mathrm{min} / 1.73 \mathrm{~m}^{2}$ & $101.8 \pm 18.6$ & $122.1 \pm 26.8$ & $<0.001$ \\
\hline $\mathrm{ACR}, \mathrm{mg} / \mathrm{g} \mathrm{Cr}$ & $9.2 \pm 26.3$ & $11.5 \pm 28.2$ & $<0.001$ \\
\hline Renal function impairment & $731(5.0)$ & $603(5.7)$ & 0.018 \\
\hline
\end{tabular}

Data are presented as mean \pm SD or $n(\%)$. BMI, body mass index; LDL, low-density lipoprotein; HDL, highdensity lipoprotein; TG, triglyceride; hsCRP, high-sensitivity C-reactive protein; BF\%, body fat percentage; eGFR, estimated glomerular filtration rate; ACR, albumin-creatinine ratio.

higher levels of BMI, waist-to-height ratio, MAP, fasting glucose, TC, TG, TG/HDL-C, hsCRP, $\mathrm{BF} \%$, and ACR, a higher prevalence of renal function impairment, and lower HDL than those with peripheral obesity and those without obesity. Among males aged 45 years or older, those with central obesity presented significantly higher BMI, waist-to-height ratio, and MAP than non-obese or peripherally obese individuals.

In both age groups, male subjects with peripheral obesity were found to exhibit a significantly better eGFR than those with central obesity and without obesity. On the other hand, younger male subjects with peripheral obesity had poorer metabolic indices (except LDL) and $\mathrm{BF} \%$, and a higher prevalence of renal function impairment than those with central obesity, whereas among older subjects, differences on metabolic indices and renal function were not significant (Table 2).

\section{Female Baseline Characteristics according to Visceral Fat Degree and BF\% Stratified by Age}

Except for the eGFR level of older women $(p=0.098)$, significant differences were found among the non-obese, peripheral, and central obese groups in all variables, and in both younger and older women. In both age groups, females with central obesity were found to have higher levels of BMI, waist-to-height ratio, MAP, fasting glucose, TG, LDL, TG/LDL, hsCRP, $\mathrm{BF} \%$, and ACR, a higher prevalence of renal function impairment, and lower levels of HDL than those with peripheral obesity and those without obesity.

Moreover, female subjects younger than 45 years with peripheral obesity presented a significantly better eGFR than those without obesity. However, among women aged 45 years 
Tsao et al.: Association between Visceral Obesity and Renal Function Impairment

Table 2. Male baseline characteristics according to visceral fat degree and percentage of body fat stratified by age

\begin{tabular}{|c|c|c|c|c|}
\hline Characteristics & Non-obese & Peripheral obese & Central obese & $p$ value \\
\hline \multicolumn{5}{|l|}{ Men $<45$ years of age } \\
\hline Number & 5,197 & 620 & 2,906 & - \\
\hline Age, years & $35.8 \pm 5.3$ & $33.5 \pm 4.9^{\mathrm{a}}$ & $37.0 \pm 5.0^{\mathrm{a}, \mathrm{b}}$ & $<0.001$ \\
\hline \multicolumn{5}{|l|}{ Measurements } \\
\hline BMI, $\mathrm{kg} / \mathrm{m}^{2}$ & $22.6 \pm 2.5$ & $24.3 \pm 1.5^{\mathrm{a}}$ & $28.1 \pm 2.5^{\mathrm{a}, \mathrm{b}}$ & $<0.001$ \\
\hline Waist-to-height ratio, $\mathrm{cm} / \mathrm{cm}$ & $0.48 \pm 0.04$ & $0.51 \pm 0.03^{\mathrm{a}}$ & $0.56 \pm 0.04^{\mathrm{a}, \mathrm{b}}$ & $<0.001$ \\
\hline Mean arterial pressure, mm $\mathrm{Hg}$ & $88.7 \pm 10.5$ & $91.9 \pm 10.1^{\mathrm{a}}$ & $96.6 \pm 11.9^{a, b}$ & $<0.001$ \\
\hline \multicolumn{5}{|l|}{ Blood chemistry data } \\
\hline Fasting glucose, $\mathrm{mmol} / \mathrm{L}$ & $5.1 \pm 1.0$ & $5.3 \pm 1.2^{\mathrm{a}}$ & $5.5 \pm 1.4^{\mathrm{a}, \mathrm{b}}$ & $<0.001$ \\
\hline Total cholesterol, mmol/L & $5.1 \pm 0.9$ & $5.3 \pm 1.0^{\mathrm{a}}$ & $5.4 \pm 1.0^{\mathrm{a}, \mathrm{b}}$ & $<0.001$ \\
\hline Triglycerides, mmol/L & $1.47 \pm 1.42$ & $1.91 \pm 2.02^{\mathrm{a}}$ & $2.34 \pm 2.51^{\mathrm{a}, \mathrm{b}}$ & $<0.001$ \\
\hline LDL-C, mmol/L & $3.2 \pm 0.8$ & $3.4 \pm 0.8^{\mathrm{a}}$ & $3.5 \pm 0.9^{\mathrm{a}}$ & $<0.001$ \\
\hline HDL-C, mmol/L & $1.25 \pm 0.28$ & $1.15 \pm 0.24^{\mathrm{a}}$ & $1.09 \pm 0.21^{\mathrm{a}, \mathrm{b}}$ & $<0.001$ \\
\hline TG/HDL-C & $1.34 \pm 2.04$ & $1.80 \pm 2.46^{\mathrm{a}}$ & $2.33 \pm 3.34^{\mathrm{a}, \mathrm{b}}$ & $<0.001$ \\
\hline hsCRP, $\mu \mathrm{g} / \mathrm{mL}$ & $1.5 \pm 4.3$ & $2.0 \pm 4.2$ & $2.6 \pm 4.9^{\mathrm{a}, \mathrm{b}}$ & $<0.001$ \\
\hline \multicolumn{5}{|l|}{ Body composition } \\
\hline $\mathrm{BF} \%$ & $18.0 \pm 4.0$ & $24.6 \pm 3.4^{\mathrm{a}}$ & $27.0 \pm 2.9^{a, b}$ & $<0.001$ \\
\hline \multicolumn{5}{|l|}{ Renal function } \\
\hline $\mathrm{eGFR}, \mathrm{mL} / \mathrm{min} / 1.73 \mathrm{~m}^{2}$ & $104.0 \pm 17.1$ & $109.9 \pm 17.8^{\mathrm{a}}$ & $105.3 \pm 19.6^{\mathrm{a}, \mathrm{b}}$ & $<0.001$ \\
\hline $\mathrm{ACR}, \mathrm{mg} / \mathrm{g} \mathrm{Cr}$ & $6.1 \pm 18.5$ & $6.8 \pm 20.0$ & $10.8 \pm 28.8^{\mathrm{a}, \mathrm{b}}$ & $<0.001$ \\
\hline Renal function impairment & $127(2.4)$ & $20(3.2)$ & $170(5.8)^{\mathrm{a}, \mathrm{b}}$ & $<0.001$ \\
\hline \multicolumn{5}{|l|}{ Men $\geq 45$ years of age } \\
\hline Number & 3,423 & 39 & 2,344 & - \\
\hline Age, years & $54.3 \pm 7.6$ & $50.1 \pm 4.9^{\mathrm{a}}$ & $54.8 \pm 8.4^{\mathrm{b}}$ & $<0.001$ \\
\hline \multicolumn{5}{|l|}{ Measurements } \\
\hline BMI, $\mathrm{kg} / \mathrm{m}^{2}$ & $22.8 \pm 2.3$ & $22.6 \pm 1.5$ & $26.8 \pm 2.4^{\mathrm{a}, \mathrm{b}}$ & $<0.001$ \\
\hline Waist-to-height ratio, $\mathrm{cm} / \mathrm{cm}$ & $0.49 \pm 0.04$ & $0.51 \pm 0.02$ & $0.56 \pm 0.04^{\mathrm{a}, \mathrm{b}}$ & $<0.001$ \\
\hline Mean arterial pressure, $\mathrm{mm} \mathrm{Hg}$ & $89.9 \pm 12.6$ & $87.6 \pm 11.5$ & $96.9 \pm 13.0^{\mathrm{a}, \mathrm{b}}$ & $<0.001$ \\
\hline \multicolumn{5}{|l|}{ Blood chemistry data } \\
\hline Fasting glucose, mmol/L & $5.5 \pm 1.4$ & $6.2 \pm 3.0^{\mathrm{a}}$ & $6.0 \pm 1.8^{\mathrm{a}}$ & $<0.001$ \\
\hline Total cholesterol, mmol/L & $5.3 \pm 1.0$ & $5.4 \pm 0.9$ & $5.4 \pm 1.0^{\mathrm{a}}$ & $<0.001$ \\
\hline Triglycerides, mmol/L & $1.52 \pm 1.33$ & $1.95 \pm 1.40$ & $2.01 \pm 1.56^{\mathrm{a}}$ & $<0.001$ \\
\hline LDL-C, $\mathrm{mmol} / \mathrm{L}$ & $3.3 \pm 0.9$ & $3.3 \pm 0.9$ & $3 . \pm 50.9^{\mathrm{a}}$ & $<0.001$ \\
\hline HDL-C, mmol/L & $1.24 \pm 0.30$ & $1.20 \pm 0.28$ & $1.11 \pm 0.24^{\mathrm{a}}$ & $<0.001$ \\
\hline TG/HDL-C & $1.39 \pm 2.01$ & $1.85 \pm 1.66$ & $1.99 \pm 2.11^{\mathrm{a}}$ & $<0.001$ \\
\hline hsCRP, $\mu \mathrm{g} / \mathrm{mL}$ & $2.1 \pm 6.9$ & $3.0 \pm 4.8$ & $2.5 \pm 4.7$ & 0.053 \\
\hline \multicolumn{5}{|l|}{ Body composition } \\
\hline $\mathrm{BF} \%$ & $18.3 \pm 3.8$ & $25.2 \pm 2.9^{\mathrm{a}}$ & $26.4 \pm 2.7^{\mathrm{a}}$ & $<0.001$ \\
\hline \multicolumn{5}{|l|}{ Renal function } \\
\hline $\mathrm{eGFR}, \mathrm{mL} / \mathrm{min} / 1.73 \mathrm{~m}^{2}$ & $97.3 \pm 18.1$ & $107.2 \pm 18.2^{\mathrm{a}}$ & $96.9 \pm 19.1^{b}$ & 0.002 \\
\hline $\mathrm{ACR}, \mathrm{mg} / \mathrm{g} \mathrm{Cr}$ & $9.1 \pm 26.2$ & $17.7 \pm 53.4$ & $14.5 \pm 36.2^{\mathrm{a}}$ & $<0.001$ \\
\hline Renal function impairment & $175(5.1)$ & $2(5.1)$ & $237(10.1)^{\mathrm{a}}$ & $<0.001$ \\
\hline
\end{tabular}

Data are presented as mean \pm SD or $n(\%)$. BMI, body mass index; LDL, low-density lipoprotein; HDL, highdensity lipoprotein; TG, triglyceride; hsCRP, high-sensitivity C-reactive protein; BF\%, body fat percentage; eGFR, estimated glomerular filtration rate; ACR, albumin-creatinine ratio. ${ }^{\mathrm{a}} p<0.05$ versus non-obese; ${ }^{\mathrm{b}} p<$ 0.05 versus peripheral obese in the Bonferroni post hoc comparisons.

or older a trend for a better eGFR was observed, although it was not significant. It is noteworthy that subjects with central obesity outperformed those with peripheral obesity and without obesity (also, subjects with peripheral obesity outperformed those without obesity) in terms of metabolism indices and BF\% (except for TC in younger female subjects) (Table 3). 
Table 3. Female baseline characteristics according to visceral fat degree and percentage of body fat stratified by age

\begin{tabular}{|c|c|c|c|c|}
\hline Characteristics & Non-obese & Peripheral obese & Central obese & $p$ value \\
\hline \multicolumn{5}{|l|}{ Women $<45$ years of age } \\
\hline Number & 2,446 & 3,465 & 72 & - \\
\hline Age, years & $33.7 \pm 5.3$ & $36.1 \pm 5.2^{\mathrm{a}}$ & $37.2 \pm 5.2^{\mathrm{a}}$ & $<0.001$ \\
\hline \multicolumn{5}{|l|}{ Measurements } \\
\hline BMI, $\mathrm{kg} / \mathrm{m}^{2}$ & $19.1 \pm 1.6$ & $22.8 \pm 2.3^{\mathrm{a}}$ & $31.9 \pm 3.6^{a, b}$ & $<0.001$ \\
\hline Waist-to-height ratio, $\mathrm{cm} / \mathrm{cm}$ & $0.43 \pm 0.03$ & $0.48 \pm 0.04^{\mathrm{a}}$ & $0.60 \pm 0.05^{a, b}$ & $<0.001$ \\
\hline Mean arterial pressure, $\mathrm{mm} \mathrm{Hg}$ & $78.6 \pm 8.8$ & $81.6 \pm 10.6^{\mathrm{a}}$ & $92.5 \pm 13.5^{\mathrm{a}, \mathrm{b}}$ & $<0.001$ \\
\hline \multicolumn{5}{|l|}{ Blood chemistry data } \\
\hline Fasting glucose, $\mathrm{mmol} / \mathrm{L}$ & $4.9 \pm 0.5$ & $5.1 \pm 0.6^{\mathrm{a}}$ & $5.4 \pm 0.8^{\mathrm{a}, \mathrm{b}}$ & $<0.001$ \\
\hline Total cholesterol, mmol/L & $4.6 \pm 0.8$ & $4.8 \pm 0.8^{\mathrm{a}}$ & $5.0 \pm 0.7^{\mathrm{a}}$ & $<0.001$ \\
\hline Triglycerides, mmol/L & $0.70 \pm 0.34$ & $0.95 \pm 0.60^{\mathrm{a}}$ & $1.44 \pm 0.77^{\mathrm{a}, \mathrm{b}}$ & $<0.001$ \\
\hline $\mathrm{LDL}-\mathrm{C}, \mathrm{mmol} / \mathrm{L}$ & $2.6 \pm 0.7$ & $2.9 \pm 0.7^{\mathrm{a}}$ & $3.3 \pm 0.6^{\mathrm{a}, \mathrm{b}}$ & $<0.001$ \\
\hline HDL-C, mmol/L & $1.56 \pm 0.31$ & $1.41 \pm 0.30^{\mathrm{a}}$ & $1.17 \pm 0.21^{\mathrm{a}, \mathrm{b}}$ & $<0.001$ \\
\hline TG/HDL-C & $0.48 \pm 0.31$ & $0.74 \pm 0.64^{\mathrm{a}}$ & $1.33 \pm 0.90^{\mathrm{a}, \mathrm{b}}$ & $<0.001$ \\
\hline hsCRP, $\mu \mathrm{g} / \mathrm{mL}$ & $0.7 \pm 1.9$ & $1.3 \pm 3.2^{\mathrm{a}}$ & $3.5 \pm 3.0^{\mathrm{a}, \mathrm{b}}$ & $<0.001$ \\
\hline \multicolumn{5}{|l|}{ Body composition } \\
\hline $\mathrm{BF} \%$ & $23.1 \pm 3.2$ & $31.8 \pm 3.5^{\mathrm{a}}$ & $44.2 \pm 5.1^{\mathrm{a}, \mathrm{b}}$ & $<0.001$ \\
\hline \multicolumn{5}{|l|}{ Renal function } \\
\hline $\mathrm{eGFR}, \mathrm{mL} / \mathrm{min} / 1.73 \mathrm{~m}^{2}$ & $127.0 \pm 24.6$ & $129.8 \pm 27.1^{\mathrm{a}}$ & $126.7 \pm 27.0$ & $<0.001$ \\
\hline $\mathrm{ACR}, \mathrm{mg} / \mathrm{g} \mathrm{Cr}$ & $8.7 \pm 21.1$ & $8.8 \pm 21.9$ & $23.9 \pm 52.4^{\mathrm{a}, \mathrm{b}}$ & $<0.001$ \\
\hline Renal function impairment & $74(3.0)$ & $121(3.5)$ & $13(18.1)^{a, b}$ & $<0.001$ \\
\hline \multicolumn{5}{|l|}{ Women $\geq 45$ years of age } \\
\hline Number & 742 & 3,378 & 458 & - \\
\hline Age, years & $54.0 \pm 7.4$ & $54.7 \pm 7.0$ & $59.3 \pm 8.1^{\mathrm{a}, \mathrm{b}}$ & $<0.001$ \\
\hline \multicolumn{5}{|l|}{ Measurements } \\
\hline BMI, $\mathrm{kg} / \mathrm{m}^{2}$ & $20.3 \pm 2.0$ & $24.1 \pm 2.2^{\mathrm{a}}$ & $29.7 \pm 2.7^{\mathrm{a}, \mathrm{b}}$ & $<0.001$ \\
\hline Waist-to-height ratio, $\mathrm{cm} / \mathrm{cm}$ & $0.47 \pm 0.05$ & $0.53 \pm 0.05^{\mathrm{a}}$ & $0.61 \pm 0.05^{\mathrm{a}, \mathrm{b}}$ & $<0.001$ \\
\hline Mean arterial pressure, $\mathrm{mm} \mathrm{Hg}$ & $84.4 \pm 12.6$ & $90.0 \pm 13.3^{\mathrm{a}}$ & $97.2 \pm 13.3^{\mathrm{a}, \mathrm{b}}$ & $<0.001$ \\
\hline \multicolumn{5}{|l|}{ Blood chemistry data } \\
\hline Fasting glucose, $\mathrm{mmol} / \mathrm{L}$ & $5.2 \pm 1.1$ & $5.5 \pm 1.3^{\mathrm{a}}$ & $6.0 \pm 1.6^{\mathrm{a}, \mathrm{b}}$ & $<0.001$ \\
\hline Total cholesterol, mmol/L & $5.3 \pm 1.0$ & $5.4 \pm 1.0^{\mathrm{a}}$ & $5.6 \pm 1.0^{\mathrm{a}, \mathrm{b}}$ & $<0.001$ \\
\hline Triglycerides, mmol/L & $0.99 \pm 0.79$ & $1.38 \pm 1.26^{\mathrm{a}}$ & $1.66 \pm 1.54^{\mathrm{a}, \mathrm{b}}$ & $<0.001$ \\
\hline LDL-C, mmol/L & $3.1 \pm 0.9$ & $3.3 \pm 0.9^{\mathrm{a}}$ & $3.5 \pm 0.9^{\mathrm{a}, \mathrm{b}}$ & $<0.001$ \\
\hline HDL-C, mmol/L & $1.53 \pm 0.32$ & $1.37 \pm 0.30^{\mathrm{a}}$ & $1.29 \pm 0.28^{\mathrm{a}, \mathrm{b}}$ & $<0.001$ \\
\hline TG/HDL-C & $0.72 \pm 0.82$ & $1.14 \pm 1.59^{\mathrm{a}}$ & $1.41 \pm 1.60^{\mathrm{a}, \mathrm{b}}$ & $<0.001$ \\
\hline hsCRP, $\mu \mathrm{g} / \mathrm{mL}$ & $1.2 \pm 4.2$ & $1.9 \pm 4.6^{\mathrm{a}}$ & $3.3 \pm 4.3^{\mathrm{a}, \mathrm{b}}$ & $<0.001$ \\
\hline \multicolumn{5}{|l|}{ Body composition } \\
\hline $\mathrm{BF} \%$ & $23.5 \pm 3.2$ & $33.4 \pm 3.6^{\mathrm{a}}$ & $42.4 \pm 3.9^{a, b}$ & $<0.001$ \\
\hline \multicolumn{5}{|l|}{ Renal function } \\
\hline $\mathrm{eGFR}, \mathrm{mL} / \mathrm{min} / 1.73 \mathrm{~m}^{2}$ & $112.5 \pm 23.4$ & $114.0 \pm 25.1$ & $111.8 \pm 28.7$ & 0.098 \\
\hline $\mathrm{ACR}, \mathrm{mg} / \mathrm{g} \mathrm{Cr}$ & $11.2 \pm 27.2$ & $14.1 \pm 32.3$ & $26.5 \pm 51.3^{\mathrm{a}, \mathrm{b}}$ & $<0.001$ \\
\hline Renal function impairment & $36(4.9)$ & $266(7.9)^{\mathrm{a}}$ & $93(20.3)^{\mathrm{a}, \mathrm{b}}$ & $<0.001$ \\
\hline
\end{tabular}

Data are presented as mean \pm SD or $n(\%)$. BMI, body mass index; LDL, low density lipoprotein; HDL, high density lipoprotein; TG, triglyceride; hsCRP, high sensitivity C-reactive protein; $\mathrm{BF} \%$, body fat percentage; eGFR, estimated glomerular filtration rate; ACR, albumin-creatinine ratios. ${ }^{\mathrm{a}} p<0.05$ versus non-obese; ${ }^{\mathrm{b}} p<$ 0.05 versus peripheral obese in the Bonferroni post hoc comparisons.

\section{Joint Effects of Age and Body Fat Distribution on Renal Function Impairment}

Prior to confounding factor adjustment, univariate (unadjusted) analysis showed a significant contribution of central obesity for renal function impairment, both in female and male subjects of all age groups. On the other hand, peripheral obesity only contributed signif- 
Table 4. Joint effects of age and body fat distribution pattern on renal function impairment in men and women

\begin{tabular}{|c|c|c|c|c|}
\hline \multirow[t]{2}{*}{ Group/type } & \multirow{2}{*}{$\begin{array}{l}\text { Patients, } \\
n\end{array}$} & \multirow{2}{*}{$\begin{array}{l}\text { Renal function } \\
\text { impairment, } \\
n(\%)\end{array}$} & \multicolumn{2}{|l|}{ OR $(95 \% \mathrm{CI})$} \\
\hline & & & model 1 & model 2 \\
\hline \multicolumn{5}{|l|}{ Men $<45$ years of age } \\
\hline Non-obese & 5,197 & $127(2.4)$ & Reference & Reference \\
\hline Peripheral obese & 620 & $20(3.2)$ & $1.33(0.82-2.15)$ & $0.86(0.51-1.46)$ \\
\hline Central obese & 2,906 & $170(5.8)$ & $2.48(1.96-3.14)^{*}$ & $1.04(0.79-1.36)$ \\
\hline \multicolumn{5}{|l|}{ Men $\geq 45$ years of age } \\
\hline Non-obese & 3,423 & $175(5.1)$ & Reference & Reference \\
\hline Peripheral obese & 39 & $2(5.1)$ & $1.00(0.24-4.20)$ & $0.80(0.18-3.62)$ \\
\hline Central obese & 2,344 & $237(10.1)$ & $2.09(1.70-2.56)^{*}$ & $1.42(1.14-1.78)^{*}$ \\
\hline \multicolumn{5}{|c|}{ Women $<45$ years of age } \\
\hline Non-obese & 2,446 & $74(3.0)$ & Reference & Reference \\
\hline Peripheral obese & 3,465 & $121(3.5)$ & $1.16(0.86-1.56)$ & $0.93(0.68-1.28)$ \\
\hline Central obese & $72^{\mathrm{a}}$ & 13 (18.1) & $7.06(3.71-13.44)^{*}$ & $3.14(1.52-6.49) *$ \\
\hline \multicolumn{5}{|c|}{ Women $\geq 45$ years of age } \\
\hline Non-obese & 742 & $36(4.9)$ & Reference & Reference \\
\hline Peripheral obese & 3,378 & $266(7.9)$ & $1.68(1.17-2.40)^{*}$ & $1.06(0.73-1.55)$ \\
\hline Central obese & 458 & $93(20.3)$ & $5.00(3.33-7.49)^{*}$ & $2.04(1.31-3.16)^{*}$ \\
\hline
\end{tabular}

Model definitions are: model 1, unadjusted analysis; model 2, adjusted for mean arterial pressure, fasting glucose and hsCRP level, fasting glucose, total cholesterol, triglycerides, LDL-C, HDL-C and TG/HDL-C. OR, odds ratio; $\mathrm{CI}$, confidence interval; hsCRP, high-sensitivity C-reactive protein; LDL, low-density lipoprotein; HDL, high-density lipoprotein; TG, triglyceride. ${ }^{*} p$ value $<0.05 .{ }^{\text {a }}$ Patient number is far less than reference.

icantly for renal function impairment in women aged 45 years or older. Following the confounding factor adjustment (including MAP, fasting glucose and hsCRP level, fasting glucose, TC, TG, LDL-C, HDL-C, and TG/HDL-C), the associations between peripheral obesity and renal function impairment were no longer observed in any of the groups. However, central obesity was identified as an independent factor of renal function impairment for all groups except males under 45 years of age (Table 4). Besides, because the patient number of central obese was far less than reference, we used traditional adjustment and compared to penalized logistic regression for odds ratio with renal function impairment outcome, finding that there was no sparse effect in the current regression models (see online suppl. material; for all online suppl. material, see www.karger.com/doi/10.1159/000496626).

\section{Discussion}

This cross-sectional study investigated the association between visceral obesity and renal function impairment in a population of 14,529 men and 10,561 women. The results showed that central obesity contributed significantly to renal function impairment, both in female and male subjects of all age groups, whereas peripheral obesity contributed significantly to renal function impairment in younger women. After adjusting for possible confounding factors, the contribution of peripheral obesity was no longer significant for any of the groups, whereas central obesity remained a significant contributing factor for renal function impairment in all groups, except men under 45 years of age.

Among the study subjects, strong gender differences were found regarding the lipid profiles, body composition, and renal function, which is consistent with the findings of 
previous studies. A previous study found that premenopausal women have a better plasma lipid profile than men, with a higher concentration of HDL-C and lower LDL, VLDL-C, and TG than age-matched men [11]. Moreover, women have more body and subcutaneous fat, particularly in the abdominal and gluteal-femoral regions, and less muscle mass and visceral fat than men [12]. A previous study revealed that although gender differences in GFR were not apparent in the age range of 20-50 years, a significant GFR decline was observed in aging males but not in females, indicating the protective role of estrogens during the premenopausal period [13]. The results of the present study are consistent with those findings. In the present study, we divided study subjects into younger and older groups because previous studies indicated that gender differences in GFR were not apparent in younger adults, but a significant decline in GFR was observed in aging male but not in female subjects [13].

Several studies have reported a correlation between visceral obesity and age, fasting glucose level, lipid profile, blood pressure, and gender, whereupon increasing age is associated with the increased accumulation of visceral fat. This trend is more obvious in women, almost quadrupling between the ages of 25 and 65 years. Men show a similar but not so dramatic trend [14]. Age strongly influences the prediction of intra-abdominal adipose tissue from the waist circumference [15]. In healthy non-obese women, the VAT area assessed through CT has been estimated to increase with age at a rate of $2.36 \mathrm{~cm}^{2}$ per year [16]. Recent studies have demonstrated that fructose consumption increases TG and glucose levels, leading to insulin resistance and exacerbating the metabolic profile presentation [17]. Moreover, fructose consumption among overweight individuals is reported to increase de novo lipogenesis, dyslipidemia, and visceral adiposity, and to decrease insulin sensitivity [18]. It has long been recognized that obese individuals present a significantly higher hypertension frequency than normal weight and underweight individuals [19]. Visceral fat releases different bioactive molecules, hormones, and proinflammatory cytokines, which trigger an increased expression of CRP [20], an acute-phase protein and a marker of systemic inflammation [21]. Therefore, visceral obesity is well recognized to be associated with low-grade inflammatory states [22]. Previous studies have reported that obese individuals present higher CRP serum concentrations than those with normal weight $[23,24]$.

VAT is known to have a high lipolytic rate, generating large amounts of free fatty acids which are delivered to the liver, increasing hepatic glucose production, hyperinsulinemia, and metabolic syndrome [5]. In contrast, the accumulation of subcutaneous adipose tissue (SAT) is independently associated with a lower risk of mortality and with certain disorders [25]. Several studies have claimed that SAT may exert protective effects [26], and recent data suggests that a high VAT/SAT ratio constitutes an independent risk factor, in addition to absolute fat volumes [27]. A study focused on the relationship between regional abdominal adiposity and insulin resistance in a group of nondiabetic, middle-aged Taiwanese subjects with varying degrees of BMI suggests that intraperitoneal fat mass (evaluated by CT) is the best predictor of insulin resistance [28]. Another systematic review reported significant correlations between most obesity indices and insulin resistance. Among these indices, VAT mass showed the strongest correlation with the homeostatic model assessment of insulin resistance, followed by total fat mass, BMI, and waist circumference [29].

A previous study reported that females are characterized by a lower VAT and a higher SAT [30]. A more pronounced VAT increase was found in men than women and in normal weight than overweight and obese individuals [31]. With regard to sex differences in central obesity (evaluated by CT), men present an almost two-fold higher VAT amount than premenopausal women [32]. Moreover, previous studies have suggested that VAT deposition significantly increases with age in both men and postmenopausal women. The decline in circulating estrogens during menopause leads to a shift in adipose tissue deposition, favoring the visceral 
depot. Previous studies have suggested that postmenopausal women may present up to twice the amount of VAT than do premenopausal women [2].

Age-associated loss of kidney function has been recognized and described in previous studies [33]. With aging, progressive decreases in eGFR and renal blood flow could be observed. Decreased glomerular capillary plasma flow rate and the glomerular capillary ultrafiltration coefficient attribute to the fall in GFR. In addition, aging is associated with altered activity and responsiveness to vasoactive stimuli. A recent study, including 3,473 subjects, found that eGFR was negatively associated with BMI, but positively related to BF. Additional roles of BMI and waist circumference were observed in subjects categorized according to BF. Normal weight obese females presented increased eGFR, whereas a higher eGFR was found in males with low/normal BF and BMI or normal waist circumference. A higher eGFR was observed in normal weight obese females in whom hyperfiltration may be suspected [34]. In the present study, eGFR was also found to be positively related to BF, especially in peripherally obese individuals. A previous cross-sectional study of Japanese adult patients with type 2 diabetes mellitus demonstrated a close association between microalbuminuria and increased visceral, but not subcutaneous, fat [35]. In the present study, after adjusting for the possible confounding factors mentioned above, visceral obesity still contributed significantly to renal function impairment in all groups.

Several mechanisms have been proposed as being responsible for the association between increased visceral fat and albuminuria. First, adipocytokine secretion abnormalities in VAT are likely to play an important role in the development of diabetic nephropathy [36]. Increased leptin secretion may contribute to renal impairment through glomerular hyperfiltration by activating the sympathetic nervous system [37] or by directly injuring the glomerular endothelial and mesangial cells [38]. Increased levels of free fatty acids, tumor necrosis factor- $\alpha$, and resistin as well as decreased levels of adiponectin may provoke renal damage via insulin resistance [36, 39]. Second, increased renin-angiotensin system activity induces renal injury as a result of increased adipose tissue synthesis [36]. Finally, increased fat in the renal hilum may compress the renal parenchyma, decreasing renal tubular flow rates, which may result in hyperfiltration via excessive proximal sodium reabsorption [36].

The present study has some limitations. First, as a cross-sectional study based on the routine health examinations of a general population, the results cannot prove a causal relationship between obesity and renal function impairment. Second, the condition of acute renal injury could not be excluded, as it may coincide with the criteria defining renal function impairment in the present study. Third, given the retrospective nature of the study, the urine ACR value could not be double-checked. Fourth, several types of anti-hypertensive medications might have affected the level of albuminuria, which could not be well adjusted due to the initial questionnaire design.

\section{Conclusion}

The present study showed that central obesity contributes significantly to renal function impairment in younger and older individuals of both sexes, except for men under 45 years of age. Although this result was significant, it cannot be easily explained, and gender differences in BF distribution and systemic sex hormone concentrations might play a significant role. However, the exact underlying mechanism remains elusive, requiring further studies. We may conclude that central obesity is independently associated with renal function impairment in all populations, except males under 45 years of age. For males under 45 years of age, with a view to preventing renal function impairment, besides decreasing $\mathrm{BF} \%$ or the level of central obesity, controlling other metabolic factors might be even more important. 


\section{Acknowledgments}

We thank the staff of the Health Management Center of Chang Gung Hospital for assistance with data collection.

\section{Statement of Ethics}

The study was approved by the Institutional Review Board of Chang-Gung Memorial Hospital and was conducted in accordance with the guidelines laid down in the Declaration of Helsinki.

\section{Disclosure Statement}

The authors declare that they have no competing interests.

\section{Funding Sources}

The authors declare that no funding was received.

\section{Author Contributions}

Y.-C.T., J.-Y.C., and W.-C.L. analyzed and interpreted the patient data and were major contributors in writing the manuscript. J.-Y.C. and W.-C.Y.interpreted the analyzed data. W.-C.L. assisted with data collection. J.-Y.C. and W.-C.L. had equal contribution to the present study as corresponding authors. All authors read and approved the final paper.

\section{References}

1 Jha V, Garcia-Garcia G, Iseki K, Li Z, Naicker S, Plattner B, et al. Chronic kidney disease: global dimension and perspectives. Lancet. 2013 Jul;382(9888):260-72.

2 Tchernof A, Després JP. Pathophysiology of human visceral obesity: an update. Physiol Rev. 2013 Jan;93(1): 359-404.

3 Després JP, Lemieux I. Abdominal obesity and metabolic syndrome. Nature. 2006 Dec;444(7121):881-7.

4 Foster MC, Hwang SJ, Larson MG, Lichtman JH, Parikh NI, Vasan RS, et al. Overweight, obesity, and the development of stage 3 CKD: the Framingham Heart Study. Am J Kidney Dis. 2008 Jul;52(1):39-48.

5 Shulman GI. Ectopic fat in insulin resistance, dyslipidemia, and cardiometabolic disease. N Engl J Med. 2014 Sep;371(12):1131-41.

6 Tamba S, Nakatsuji H, Kishida K, Noguchi M, Ogawa T, Okauchi Y, et al. Relationship between visceral fat accumulation and urinary albumin-creatinine ratio in middle-aged Japanese men. Atherosclerosis. 2010 Aug; 211(2):601-5.

7 Mulyadi L, Stevens C, Munro S, Lingard J, Bermingham M. Body fat distribution and total body fat as risk factors for microalbuminuria in the obese. Ann Nutr Metab. 2001;45(2):67-71.

8 Ma YC, Zuo L, Chen JH, Luo Q, Yu XQ, Li Y, et al. Modified glomerular filtration rate estimating equation for Chinese patients with chronic kidney disease. J Am Soc Nephrol. 2006 Oct;17(10):2937-44.

9 Keane WF, Eknoyan G. Proteinuria, albuminuria, risk, assessment, detection, elimination (PARADE): a position paper of the National Kidney Foundation. Am J Kidney Dis. 1999 May;33(5):1004-10.

10 Levey AS, Coresh J, Balk E, Kausz AT, Levin A, Steffes MW, et al.; National Kidney Foundation. National Kidney Foundation practice guidelines for chronic kidney disease: evaluation, classification, and stratification. Ann Intern Med. 2003 Jul;139(2):137-47.

11 Wang X, Magkos F, Mittendorfer B. Sex differences in lipid and lipoprotein metabolism: it's not just about sex hormones. J Clin Endocrinol Metab. 2011 Apr;96(4):885-93.

12 Ethun K. Sex and gender differences in body composition, lipid metabolism, and glucose regulation. In: Neigh G, Mitzelfelt M, editors. Sex differences in physiology. New York: Elsevier Science; 2016. pp. 145-65.

13 Sabolić I, Asif AR, Budach WE, Wanke C, Bahn A, Burckhardt G. Gender differences in kidney function. Pflugers Arch. 2007 Dec;455(3):397-429. 
Tsao et al.: Association between Visceral Obesity and Renal Function Impairment

14 Hunter GR, Gower BA, Kane BL. Age Related Shift in Visceral Fat. Int J Body Compos Res. 2010 Sep;8(3):103-8.

15 Han TS, McNeill G, Seidell JC, Lean ME. Predicting intra-abdominal fatness from anthropometric measures: the influence of stature. Int J Obes Relat Metab Disord. 1997 Jul;21(7):587-93.

16 DeNino WF, Tchernof A, Dionne IJ, Toth MJ, Ades PA, Sites CK, et al. Contribution of abdominal adiposity to age-related differences in insulin sensitivity and plasma lipids in healthy nonobese women. Diabetes Care. 2001 May;24(5):925-32.

17 Teff KL, Grudziak J, Townsend RR, Dunn TN, Grant RW, Adams SH, et al. Endocrine and metabolic effects of consuming fructose- and glucose-sweetened beverages with meals in obese men and women: influence of insulin resistance on plasma triglyceride responses. J Clin Endocrinol Metab. 2009 May;94(5):1562-9.

18 Stanhope KL, Schwarz JM, Keim NL, Griffen SC, Bremer AA, Graham JL, et al. Consuming fructose-sweetened, not glucose-sweetened, beverages increases visceral adiposity and lipids and decreases insulin sensitivity in overweight/obese humans. J Clin Invest. 2009 May;119(5):1322-34.

19 Stamler R, Stamler J, Riedlinger WF, Algera G, Roberts RH. Weight and blood pressure. Findings in hypertension screening of 1 million Americans. JAMA. 1978 Oct;240(15):1607-10.

20 Zelkha SA, Freilich RW, Amar S. Periodontal innate immune mechanisms relevant to atherosclerosis and obesity. Periodontol 2000. 2010 Oct;54(1):207-21.

21 Palmer BF, Clegg DJ. The sexual dimorphism of obesity. Mol Cell Endocrinol. 2015 Feb;402:113-9.

22 Yudkin JS, Stehouwer CD, Emeis JJ, Coppack SW. C-reactive protein in healthy subjects: associations with obesity, insulin resistance, and endothelial dysfunction: a potential role for cytokines originating from adipose tissue? Arterioscler Thromb Vasc Biol. 1999 Apr;19(4):972-8.

23 Moradi S, Mollabashi M, Kerman SR. Relation between C-reactive protein and body mass index in patients with polycystic ovarian syndrome. Gynecol Endocrinol. 2011 Jul;27(7):480-5.

24 Phelan N, O'Connor A, Kyaw Tun T, Correia N, Boran G, Roche HM, et al. Leucocytosis in women with polycystic ovary syndrome (PCOS) is incompletely explained by obesity and insulin resistance. Clin Endocrinol (Oxf). 2013 Jan;78(1):107-13.

25 Tankó LB, Bagger YZ, Alexandersen P, Larsen PJ, Christiansen C. Central and peripheral fat mass have contrasting effect on the progression of aortic calcification in postmenopausal women. Eur Heart J. 2003 Aug; 24(16):1531-7.

26 Porter SA, Massaro JM, Hoffmann U, Vasan RS, O’Donnel CJ, Fox CS. Abdominal subcutaneous adipose tissue: a protective fat depot? Diabetes Care. 2009 Jun;32(6):1068-75.

27 Kaess BM, Pedley A, Massaro JM, Murabito J, Hoffmann U, Fox CS. The ratio of visceral to subcutaneous fat, a metric of body fat distribution, is a unique correlate of cardiometabolic risk. Diabetologia. 2012 Oct;55(10): 2622-30.

28 Hsieh CJ, Wang PW, Chen TY. The relationship between regional abdominal fat distribution and both insulin resistance and subclinical chronic inflammation in non-diabetic adults. Diabetol Metab Syndr. 2014 Apr;6(1): 49.

29 Zhang M, Hu T, Zhang S, Zhou L. Associations of Different Adipose Tissue Depots with Insulin Resistance: A Systematic Review and Meta-analysis of Observational Studies. Sci Rep. 2015 Dec;5(1):18495.

30 Machann J, Thamer C, Schnoedt B, Stefan N, Stumvoll M, Haring HU, et al. Age and gender related effects on adipose tissue compartments of subjects with increased risk for type 2 diabetes: a whole body MRI/MRS study. MAGMA. 2005 Jul;18(3):128-37.

31 Bouchard C, Després JP, Mauriège P. Genetic and nongenetic determinants of regional fat distribution. Endocr Rev. 1993 Feb;14(1):72-93.

32 Kvist H, Chowdhury B, Grangård U, Tylén U, Sjöström L. Total and visceral adipose-tissue volumes derived from measurements with computed tomography in adult men and women: predictive equations. Am J Clin Nutr. 1988 Dec;48(6):1351-61.

33 Weinstein JR, Anderson S. The aging kidney: physiological changes. Adv Chronic Kidney Dis. 2010 Jul;17(4): 302-7.

34 Tsai YW, Ho CI, Chen JY, Chang KC, Weng YM, Chen SY, et al. Impact of body composition on estimated glomerular filtration rate in relatively healthy adults in Taiwan. Eur J Clin Nutr. 2015 Jan;69(1):34-9.

35 Hanai K, Babazono T, Nyumura I, Toya K, Ohta M, Bouchi R, et al. Involvement of visceral fat in the pathogenesis of albuminuria in patients with type 2 diabetes with early stage of nephropathy. Clin Exp Nephrol. 2010 Apr; 14(2):132-6.

36 Wahba IM, Mak RH. Obesity and obesity-initiated metabolic syndrome: mechanistic links to chronic kidney disease. Clin J Am Soc Nephrol. 2007 May;2(3):550-62.

37 Alvarez GE, Beske SD, Ballard TP, Davy KP. Sympathetic neural activation in visceral obesity. Circulation. 2002 Nov; 106(20):2533-6.

38 Wiecek A, Kokot F, Chudek J, Adamczak M. The adipose tissue-a novel endocrine organ of interest to the nephrologist. Nephrol Dial Transplant. 2002 Feb;17(2):191-5.

39 Parvanova AI, Trevisan R, Iliev IP, Dimitrov BD, Vedovato M, Tiengo A, et al. Insulin resistance and microalbuminuria: a cross-sectional, case-control study of 158 patients with type 2 diabetes and different degrees of urinary albumin excretion. Diabetes. 2006 May;55(5):1456-62. 